\title{
Selected rat strains HT, LT as a model for the study of dysadaptation states dependent on the level of excitability of the nervous system
}

\author{
N.A. Dyuzhikova*, A.I. Vaido \\ Pavlov Institute of Physiology of the RAS, St. Petersburg, Russia \\ *e-mail:dyuzhikova@mail.ru
}

Key words: selection, excitability, rat strains, stress, anxiety disorders

To elucidate the interconnections between the functional state of the nervous system (excitability), the functioning of the brain and a wide range of behavioral characteristics, a selection program with a primary goal of generating rat strains differing in thresholds of excitability of the nervous system has been launched in the 70s of the last century [1]. Wistar rats (Stolbovaya) were used as a starting material. The selection has been carried on the magnitude of the threshold of electric excitability (rectangular electric impulses, $2 \mathrm{~ms}$ ) of the tibial nerve (n. tibialis). Four rat strains with different gradation of excitability thresholds have been breeded: HT1, LT1, HT2, LT2 (high and low thresholds, 1, 2 - numbers of breeding programs) [1]. Currently, two strains that have passed more than 70 generations of breeding are maintained - HT1 (HT) and LT2 (LT) having mostly contrasting thresholds of excitability $(3.5 \pm 0.3$ and $0.70 \pm 0.04 \mathrm{~V}$, respectively). Over this long research period, the rats of these strains have been manifesting differences not only in tibial nerve excitability, but also in the thresholds of excitability in other parts of the nervous system, both the peripheral and CNS (mainly subcortical structures). The impact of the nervous system excitability on a wide spectrum of conditioned and unconditioned behavioral characteristics has been revealed [2]. In these rat strains, alterations have been detected in various parts of hormonal regulation systems, neurotransmission, the ion channels functioning, structural and functional properties of the nerve cell membranes. Strains have manifested different stress-reactivity in tests of sleep deprivation, immobilization, response to short and long-term emotional-painful stress. Prolonged post-stress behavioral manifestations persist for 6 months in the both HT and LT rats. Disorders of higher nervous activity have strain-specific manifestations: formation of a depressive-like behavior in the low-excitable HT strain, an increase in excitability, aggression, the disturbance of plastic processes, and an increase in stereotypic behavior ( jactatio capitis) in the highly excitable LT strain. These characteristics allow the usage of these strains as model objects for studies on the post-stress anxiety disorders, in particular, post-traumatic stress disorder (PTSD) and compulsive disorder (CD). The long-term effects of stress are based on the morphological changes of neurons in various brain structures, differential chromatin modifications in neurons and other somatic cells associated with epigenetic DNA and histone modifications $[3,4]$. The horizons of using the HT and LT strains for the elucidating genetic and epigenetic mechanisms of dysadaptation in response to environmental factors in terms of a personified medicine with a glance on the nervous system excitability are discussed. Acknowledgements: Supported by Presidium of UB RAS (0134-2018-0003).

\section{References}

1. Vaido A.I., Sitdikov M.Kh. (1979) Selection of rat strains by long-term threshold of excitability of neuromuscular apparatus. Genetica. XV(1):144-148.

2. Levina A.S. et al. (2018) Comparative behavioral characteristics of two rat strains differing in the nervous system excitability threshold in a spatial learning task in the Morris water maze. Zh. Vyssh. Nerv. Deiat im I.P. Pavlova. 68(2):1-12.

3. Vaido A.I. et al. (2009) Systemic control of the molecular, cell and epigenetic mechanisms of long-lasting consequences of stress. Genetica. 45(3):342-348.

4. Dyuzhikova N.A. et al. (2015) Epigenetic mechanisms in post-stress states. Usp Fiziol Nauk. 46(1):47-75. 